Horizons philosophiques

\title{
L'impossible Europe « sociale " dans le cadre de la mondialisation actuelle
}

\section{Riccardo Petrella}

Volume 11, numéro 2, printemps 2001

L’Europe, le laboratoire d'idées du XXI ${ }^{\mathrm{e}}$ siècle?

URI : https://id.erudit.org/iderudit/801178ar

DOI : https://doi.org/10.7202/801178ar

Aller au sommaire du numéro

Éditeur(s)

Collège Édouard-Montpetit

ISSN

1181-9227 (imprimé)

1920-2954 (numérique)

Découvrir la revue

Citer cet article

Petrella, R. (2001). L’impossible Europe « sociale » dans le cadre de la mondialisation actuelle. Horizons philosophiques, 11(2), 6-28.

https://doi.org/10.7202/801178ar d'utilisation que vous pouvez consulter en ligne.

https://apropos.erudit.org/fr/usagers/politique-dutilisation/ 


\section{L'IMPOSSIBLE EUROPE «SOCIALE» DANS LE CADRE DE LA MONDIALISATION ACTUELLE}

\section{INTRODUCTION.}

On s'interroge de plus en plus dans les pays de l'Union européenne sur la signification de la portée réelle du concept de "politique sociale" à la lumière du changement de paradigme idéologique (politicoéconomique) intervenu à partir des années ' 80 . La thèse soumise ici au débat est la suivante.

Nous avons assisté en Europe occidentale, dans le cadre des évolutions communes au «monde global» occidental, à une privatisation du politique et à une marchandisation généralisée de la vie et du social.

La privatisation du politique s'est affirmée par le transfert des pouvoirs de décision en matière d'allocation des ressources disponibles et de redistribution de la richesse produite opéré en faveur de sujets privés (les détenteurs du capital privé et les entreprises) à l'initiative ou avec le consentement des sujets publics (parlements, gouvernements, autorités publiques locales). La privatisation du politique a eu lieu grâce aux mesures de libéralisation des marchés, de dérégulation de l'État et des activités socio-économiques, de privatisation de pans entiers de l'économie, de la compétitivité tous azimuts. Tout cela a conduit dans nos sociétés à la primauté du capital financier en tant que paramètre principal et de définition de la valeur.

La marchandisation de la vie a progressé ces dernières années à pas de géant, en particulier, à la généralisation du Droit de Propriété Intellectuelle dont l'application a été étendue massivement au domaine de l'information et de la communication (les logiciels, par exemple) et du vivant. L'éducation est de plus en plus un domaine marchand. Depuis peu, les gènes humains peuvent être brevetés pour des buts thérapeutiques et faire l'objet de transactions commerciales. Dans de nombreux pays, l'eau - source principale de vie insubstituable, avec l'air - est tombée sous la propriété et/ou la gestion des entreprises privées pour qui l'eau-vie n'est qu'une marchandise, de plus en plus très rentable, au même titre que le pétrole ou les bananes.

Dès lors, les pays de l'Union européenne donnent l'impression qu'ils se trouvent dans une phase historique de la construction de l'Europe où ce qui prédomine est la logique de l'organisation d'un marché unique continental qui se veut très ouvert aux marchés mondialisés et d'une 
monnaie unique dont la détermination de la valeur et sa stabilité sont laissées aux marchés financiers mondiaux.

Dans ce contexte, le "social» est devenu, par rapport au capital et à l'économique (et donc au financier, au technologique....), une dimension secondaire, externe au cœur de la décision politique. Le "social» ne définit plus les priorités du politique. La politique sociale européenne a été réduite à un ensemble de mesures destinées :

- D'une part, à traiter les "maladies" dont peut souffrir la société, notamment dans une phase de changements rapides et multiples comme l'actuelle. On pense ici aux inadaptations sociales, aux anomalies sociales, aux inégalités croissantes, aux phénomènes d'exclusion, à la violence.... On reste dans le champ traditionnel de l'assistance sociale, de la compassion envers les perdants, ceux qui ne parviennent pas à s'adapter...

- D'autre part, à promouvoir la capacité des individus et des groupes sociaux, notamment à risque ou en état de marginalisation, à s'intégrer dans et s'adapter au systeme actuel (c'est ce que l'on appelle le champ nouveau de la politique de ('’État social actif»).

Ainsi, la politique sociale européenne actuelle n'échappe pas à l'emprise de la logique marchande et financière du capital privé. Est-il possible de définir et mettre en œuvre une autre politique sociale européenne? Certainement, mais à condition qu'on abandonne les principes inspirateurs et organisateurs de l'économie capitaliste de marché aujourd'hui prédominante.

\section{PRINCIPES INSPIRATEURS ET CARACTÉRISTIQUES MAJEURES DE L'ÉCONOMIE ACTUELLE.}

Telle que nous la connaissons aujourd'hui, l'économie capitaliste de marché a commencé à (ré)acquérir, au milieu des années '60, le pouvoir de gouverne et de contrôle de l'allocation des ressources matérielles et immatérielles disponibles de nos pays. D'abord, en Union soviétique, suite à la dénonciation, en 1954 par Nikita Khroutchev, des dévastations de l'économie "socialiste" opérées par le stalinisme et l'appel à une réforme radicale de l'économie soviétique centrée sur l'ouverture aux règles de marché de l'économie libérale. Ensuite, aux États-Unis, où la première réforme du Welfare State, 30 ans après sa naissance, réalisée sous le Président démocrate L.B. Johnson à l'enseigne de "war on povertys, s'est traduite par l'accentuation de la dualisation du système de Welfare américain entre, d'une part, la sécurité "sociale" assurée par le marché sur base privée (via les plans personnels d'entreprise et les assurances privées) et, d'autre part, l'assistance sociale (l'aide publique) 
octroyée à ceux et celles qui sortent du «marché».

Depuis, l'histoire économique et politico-sociale de nos pays a été caractérisée par une prise de pouvoir rapide - à partir des années ' 80 par des conceptions et des politiques qui ont fait des valeurs et des critères propres à l'économie de marché capitaliste les valeurs et les critères exclusifs de référence et de mesure de ce qui est bon, utile et nécessaire en économie comme ailleurs.

\section{L'inévitabilité supposée du marché mondial autorégulateur}

Ces conceptions se basent sur l'idée - que les forces politicosociales et économiques porteuses de ces conceptions ont réussi à faire accepter auprès de nos populations - que le sens actuel de l'histoire des sociétés contemporaines est celui de la constitution inexorable et inévitable d'un grand marché mondial unique, intégré, autorégulateur ("The Single Global Market Place»). Telle serait la destinée logique, "naturelle" de l'économie, en raison également des évolutions technologiques en matière de transport, d'information et de communication qui auraient transformé le monde en un «village global»1.

Freiner, voire empêcher ou se mettre "contre" cette évolution est considéré faire acte d'aveuglement, d'inconscience, signifie se mettre "en dehors" de l'histoire. L'avènement du marché mondial unique, intégré, autorégulateur, est - affirme-t-on - dans le cours de l'histoire. On ne peut pas l'éviter, on ne saurait pas y résister.

On comprend, dès lors, pourquoi les classes dirigeantes de nos pays ont estimé, ces 20 dernières années notamment, que leur rôle principal en tant que pouvoirs publics - était de faciliter les processus portants vers la constitution du marché mondial et de créer - chacun dans son pays les conditions les plus favorables pour que l'intégration/adaptation du marché local (national) dans le marché mondial se fasse de la manière la plus efficace, dans l'intérêt prioritaire des agents économiques opérant sur le marché local.

Le vécu quotidien des années ' 80 et '90 le confirme : les États nationaux se sont donné comme tâche principale celle de promouvoir la meilleure intégration possible de l'économie nationale dans l'économie mondiale. Tout est subordonné à cette finalité : la politique d'investissement, l'innovation technologique, la politique du marché du travail, l'enseignement et la formation, les règles du commerce, la politique fiscale et, en Europe occidentale, l'intégration européenne ${ }^{2}$ des 15 dernières

1. Une autre image, plus récente, liée toujours aux nouvelles technologies d'information et de communication, est celle du "réseau mondial auto-organisé".

2. Cf. R. PETRELLA, "La dépossession de l'État", dans Le Monde diplomatique (août 1999). 
années a été une illustration des plus éclairantes de cette subordination ${ }^{3}$.

\section{La technologie asservie aux besoins de l'industrie}

À partir des années '70, la technologie a pris clairement une portée et une signification de plus en plus "stratégiques" au fur et à mesure que la science (la connaissance) devenait l'un des facteurs-clé de la production et de la redistribution de la richesse.

Inspirée par des conceptions néo-schumpeteriennes alimentées par la croyance, à la Kondratieff, que nos économies allaient entrer dans la phase de démarrage d'un nouveau cycle long de développement axé sur les nouvelles technologies de l'information et de la communication, la politique technologique est devenue l'instrument principal qui a scellé une nouvelle alliance entre les pouvoirs publics et les «libres forces du marché" (les entreprises privées) dans leur commune bataille pour réussir l'intégration compétitive de l'économie nationale dans l'économie mondiale.

Cette alliance s'est traduite concrètement par la stratégie du développement technologique au service des "champions nationaux". L'inspiration de base de la politique technologique publique et privée a été la prédominance donnée à la politique de l'offre technologique : offre en terme d'infrastructures (recherche fondamentale, meilleure relation université-industrie, bases de données, systèmes d'information et de communication, transports, formation universitaire) ainsi qu'en termes de processus de production et de produits/services améliorés sur le plan du prix, de la qualité, de la variété. Le choix en faveur de la politique d'offre est tout à fait "naturel» dans le cadre du choix opéré en amont en faveur de la politique "inévitable" de l'intégration compétitive de l'économie nationale dans l'économie mondiale. En effet, si l'objectif est de permettre aux industries du pays d'être compétitives sur les divers marchés locaux solvables en voie de mondialisation, on ne peut que donner priorité à l'offre d'outils plus performants capables, à court terme, de répondre aux besoins du marché solvable 4 et d'agrandir la quote-part du marché, quelles que soient les conséquences à moyen et à long termes sur le plan économique global, social, politique et culturel.

On a assisté ainsi, à côté des stratégies en faveur des champions nationaux (sorte de néo-nationalisme technologique à l'échelle mondiale),

3. M. FARRELL, Integration and Inequality. The institutional limitations of European Union technological policy. Communication présentée à la conférence EAEPE à Prague, 5-7 novembre 1999.

4. Cf. R. PETRELLA, "Technological Innovation and Welfare", dans Concepts and Transformations, vol. 3, no 3 (1998), p. 283-298. Rappelons que, en règle générale, les besoins du marché solvable ne représentent pas la totalité des besoins de la société. 
à I 'émergence d'une politique de "techno-globalisme" exprimant le passage de l'intégration/adaptation à l'intégration/conquête. Apparemment différentes voies contradictoires, ces deux phénomènes représentent les deux faces de la même médaille : les marchés, les économies se mondialisent grâce, entre autres, aux développements technologiques. Dès lors, il appartient aux pouvoirs nationaux de faire réussir la conquête de ces marchés, et faire grandir la puissance des entreprises "du pays" par une politique technologique volontariste, compétitive, "agressive». Notons au passage que cette politique de l'offre technologique a instrumentalisé à ses fins le système d'éducation secondaire et supérieure universitaire et post-universitaire, dont désormais la tâche est devenue celle de former la ressource humaine qualifiée dont ont besoin les entreprises du pays pour réussir favorablement leur intégration/conquête dans le marché mondial.

\section{La formation du capitalisme mondial}

L'avènement du marché mondial intégré ayant été posé comme un "fait» inévitable (sorte de dogme, donc indiscutable), on comprend également l'importance des trois principes idéologiques - sorte de nouvelle "Sainte Trinité" - qui ont remodelé le système économique et politico-social de nos pays au cours des 20 dernières années, à savoir :

- la libéralisation des marchés

- la déréglementation de l'économie

- Ola privatisation de pans entiers de l'économies.

La libéralisation des marchés, inscrite dans les politiques qui, après la $2^{\theta}$ guerre mondiale ont défini le GATT et le système de Bretton Woods (Fond Monétaire International et Banque mondiale), est devenue «inévitable» suite à la libéralisation des mouvements de capitaux in et out des États-Unis, décidée en 1974 par Nixon, après que celui-ci ait mis fin, en 1971, à la convertibilité du dollar (plongeant ainsi l'économie mondiale dans une grande instabilité monétaire). $\hat{A}$ la fois symbolique et pragmatique, la libéralisation des mouvements de capitaux, qui s'est répandue partout dans le monde, a donné naissance en 15 ans à la formation du marché capitaliste mondial, marqué par la quasi liberté de mouvement du capital à travers le monde, avec quelques petits bémols réintroduits suite aux dévastations produites par la crise du système financier de 1997, notamment dans les pays du Sud-est asiatique.

Cela explique les raisons pourquoi la liberté du marché est désormais considérée comme l'expression fondamentale de la liberté dans nos pays.

5. Sur ces trois "enzymes" de la mondialisation actuelle, voir Groupe de Lisbonne, Les Limites de la Compétitivité, Bruxelles, Éd. Labor; Paris, La Découverte; Montréal, Boréal, 1995. 
De la liberté de marché, affirme-t-on, dépendent toutes les autres libertés. Toute limitation à la liberté du marché se traduirait, nous dit-on, par des limitations aux autres libertés (telle que la liberté de la propriété, la liberté d'entreprendre, la liberté du commerce, la liberté d'innover...). La libéralisation des mouvements des capitaux a, tout naturellement, encouragé et renforcé les processus de libéralisation des autres marchés. A maints égards, première étape avant la déréglementation et la privatisation, la libéralisation est de plus en plus poursuivie dans tous les domaines et «imposée» à tout pays qui, autrement, risque d'être accusé de "protectionnisme" et donc d'être mis ou de rester au ban de l'économie mondiale (voir les conditions mises par les pays occidentaux à l'aide donnée aux pays de l'ex-Union Soviétique et l'Europe de l'Est conformément à la politique traditionnelle du FMl et de la Banque mondiale).

Les succès réalisés dans la libéralisation des marchés industriels, agricoles, voire des services (y compris le domaine culturel...) a donné une forte impulsion au processus de déréglementation du fonctionnement de l'économie. On ne veut plus laisser à l'État que le rôle défini dans la nouvelle alliance (faciliter l'intégration de l'économie locale dans l'économie mondiale). Pour le reste, il faut dé-inventer l'État, comme l'a proposé The Economist le 20 mai 1995. II faut que l'État laisse aux forces du marché la régulation de l'économique. C'est aux capitaux organisés et circulant librement à l'échelle mondiale que revient la fonction régulatrice de l'économie mondiale. Pour que les marchés financiers mondiaux exercent, de manière efficace, une telle fonction, nos économies ont été l'objet d'une prise en propriété privée systématique, grâce aux processus massifs et rapides de privatisations de tout ce qui est privatisable. Tout y passe : les banques, les assurances, les avions, les trains, les transports urbains, l'eau, le gaz, l'électricité, les hôpitaux, les universités, l'école, la formation professionnelle.

\section{La (dé)régulation financière a remplacé la régulation politique}

En effet, la monnaie a cessé d'être principalement un moyen aux mains des pouvoirs publics nationaux pour orienter et manœuvrer l'économie nationale en fonction des objectifs de développement humain et social économiquement sain, juste et efficace. La monnaie est devenue surtout une marchandise comme toute autre marchandise, que l'on achète et que l'on vend sur les marchés financiers mondiaux à la recherche d'une plus grande value. Ce sont les opérateurs/gestionnaires des capitaux, circulant librement dans les différentes places financières, qui déterminent, en une très large mesure, la valeur des monnaies/marchandises. Le contrôle de la monnaie a échappé, de manière significative, aux pouvoirs politiques (les Parlements notamment) 
au bénéfice des marchés financiers. Ces derniers ne font que proclamer que ce déplacement de pouvoirs est normal et juste, car les marchés financiers sont supposés fonctionner de manière plus "rationnelle» que les États. Sur base d'une telle présomption (non confortée par la réalité), ils se croient autorisés à imposer ce qu'ils appellent la «discipline financière" aux autorités politiques nationales et, bien entendu, aux citoyens/consommateurs. La transformation de la monnaie en marchandise échangée sur les marchés financiers mondiaux s'est traduite, dans une série en cascade d'impératifs économiques tels que :

- inflation zéro,

- balance des paiements équilibrée,

- équilibres budgétaires et donc réduction des déficits publics, voire depuis 1998, politique des surplus budgétaires

- réduction des dépenses publiques, en particulier des dépenses sociales,

- réduction de la pression fiscale sur le capital et incitations fiscales en faveur desinvestissements privés.

Tout cela dans le but de permettre à une monnaie d'être une monnaie "forte" et aux actions et obligations du pays d'acquérir, par ce biais et par une compétitivité accrue, une plus grande valeur vis-à-vis des opérateurs des marchés financiers. Dans ces conditions, l'affirmation du Président de la Bundesbank, le 3 février 1996 à Davos (Suisse) au World Economic Forum, que «les dirigeants politiques doivent savoir qu'ils sont désormais soumis au contrôle des marchés financiers" ne fait que refléter un état de chose objectif.

La financiérisation de la régulation économique opère dans un contexte marqué de plus en plus par une nette dissociation entre économie financière et économie réelle. Sur les deux mille milliards de \$ USA que représentent les transactions financières quotidiennes actuelles à l'échelle mondiale, on estime (elles s'élevaient à 1,4 mille milliards au début de cette décennie, d'après une étude de la Banque d'Angleterre d'octobre 1995) que $10 \%$ seulement de ces transactions sont destinées à financer la création de nouvelles richesses. Le reste est de nature spéculative. C'est de la prédation : on prend la richesse là où elle existe, en jouant précisément sur les différences de prix réels et anticipés des diverses monnaies.

Un autre aspect important de la financiérisation de l'économie et qui caractérise ainsi l'économie de marché capitaliste mondialisée, libéralisée, déréglementée, privatisée, est représenté par la financiérisation des entreprises de production de biens et de services. Être compétitif pour 
rester dans la course à l'innovation technologique et à la conquête des marchés coûte de plus en plus cher, même pour les grandes entreprises multinationales. D'où, chaque année, la vague croissante de fusions, méga-fusions, acquisitions minoritaires ou majoritaires, et alliances, qui font du marché mondial actuel un marché à forte tendance oligopoliste voire quasi monopoliste, tout le contraire du marché concurrentiel autorégulateur. D'où, aussi, suite à la réduction massive du rôle d'intermédiation financière jadis joué par les banques, la tendance des entreprises à jouer simultanément sur les avoirs industriels et les avoirs financiers, l'importance de ces derniers prenant, ces dernières années, la primauté sur les stratégies industrielles proprement dites. Comme on le dit partout, c'est désormais le financier qui, dans l'entreprise, exerce la fonction de véritable PDG.

\section{Le commerce mondial : régulateur à toutes les sauces.}

Comme si la régulation financière n'était pas suffisante, voilà, depuis quelques années, le commerce élevé au rang de deuxième volant principal de la régulation de l'économie mondiale, un rôle qui a été consacré en 1995 par la création de l'OMC (Organisation Mondiale du Commerce) dotée d'importants pouvoirs de juridiction et de sanctions.

Fort heureusement, le pouvoir de l'OMC vient de recevoir un coup sévère que j'espère effectif et durable, à Seattle où les négociations dites du "cycle du Millénaire" se sont terminées par un échec retentissant sous la pression de l'opinion publique exprimée par la mobilisation à travers le monde, et non seulement à Seattle, de la société civile contre l'OMC, ses règles et ses plans de libéralisation généralisée de l'économie mondiale. Selon les promoteurs de la conférence intergouvernementale de l'OMC accueillie à Seattle du 30 novembre au 4 décembre 1999, les «négociations du Millénaire" auraient dû permettre l'élimination de toutes les barrières au commerce des biens et des services (y compris les domaines de l'éducation et de la santé), ainsi que le renforcement et l'extension des droits de propriété intellectuelle. La liberté du commerce aurait dû primer sur toute autre régulation qui, pour des raisons sociales, environnementales, économiques et culturelles mettrait des limites au commerce. Si cette règle, déjà appliquée par l'OMC dans plusieurs cas (celui du commerce des bananes, par exemple) l'avait emporté à Seattle, la souveraineté des États, des pouvoirs publics, des parlements en matière de sécurité alimentaire, de politique gouvernementale et de droits sociaux en aurait été gravement amputée. Fort heureusement, les négociations ont échoué devant le refus de l'opinion publique mondiale de réduire le monde et la vie à une marchandise et à un "grand marché général mondial» où les plus forts pourraient imposer leurs intérêts. 
Les manifestations populaires ont dit aux "puissants» du monde actuel qu'on ne peut pas confier au commerce le rôle de régler le développement social, humain et démocratique de la planète.

\section{La compétitivité en guise de légitimation}

Face aux bouleversements, jusqu'ici hélas trop rapidement et sommairement esquissés, les dirigeants de l'économie de marché capitaliste ont été obligés de porter des raisons de légitimation d'une telle évolution. Ce fut chose faite par l'impératif de la compétitivité élevée au rang de but principal des stratégies des agents économiques privés et publics. Grâce à une multitude, sans cesse croissante au cours des 20 dernières années, en toutes les langues du monde, de rapports d'experts, d'études académiques, de conférences nationales, continentales et mondiales entre le top du business, des universités, des pouvoirs publics et, grâce, aussi, aux nombreux comités nationaux, européens et mondiaux chargés de suivre les problèmes de la compétitivité, le culte de ce nouvel évangile a été prêché, divulgué, voire imposé aux populations des pays riches et puissants du monde comme à celles des pays faibles, pauvres et désavantagés. "C'est parce que je suis l'entreprise la plus compétitive que j'ai le droit, après avoir conquis le pouvoir dans le marché mondial, de gouverner la destinée de mon secteur d'activité. II en va de même des entreprises prises ensemble : celles qui survivent ont démontré qu'elles sont excellentes. Dès lors, elles ont le droit légitime de gouverner l'économie mondiale".

Réduit à une série de marchés libéralisés et déréglementés à conquérir, le monde est devenu le théâtre privilégié des opérations de guerre technologique, industrielles, commerciales entre les groupes mondiaux d'entreprises multinationales.

\section{LES DÉVASTATIONS DE L'ÉCONOMIE ACTUELLE}

Depuis le début des années ' 80 , de plus en plus nombreuses ont été les voix qui se sont élevées pour dénoncer les insuffisances et les dérives auxquelles allait conduire le choix opéré en faveur de la priorité à donner à l'intégration/adaptation de l'économie nationale (subordonnée aux intérêts des entreprises les plus puissantes du pays) dans l'économie mondiale laissée aux mains des «libres» forces du marché. Aujourd'hui, on ne peut plus nier les profondes dévastations qui touchent aux fondements mêmes de nos sociétés. 


\section{L'État aux orties : recul de l'acteur public, suprématie} grandissante des acteurs privés. La démocratie politique en danger.

Le résultat de la mise en pratique des principes de la Sainte Trinité de la Libéralisation des marchés, de la Déréglementation et de la Privatisation de l'économie a été très clair : partout on a démantelé ou on a réduit de manière significative le pouvoir d'orientation et de contrôle des acteurs publics, à commencer par les parlements, expression centrale de la représentation politique des citoyens en régime démocratique. Les parlements ne fixent plus l'ordre du jour et des priorités de leur pays. L'ordre du jour est fixé par les acteurs privés et en particulier par les marchés financiers. Cet ordre du jour devient, pour les pouvoirs publics, une contrainte considérée "exogène", à laquelle ils ne peuvent que s'adapter. Le discrédit du politique (non seulement du politique "national») qui a été promu et facilité par la scolastique des «libres» forces du marché, prétextant que la façon la meilleure pour réussir l'intégration/adaptation de l'économie nationale dans l'économie mondiale est de déréglementer, privatiser et libéraliser, est aujourd'hui fort répandu parmi les populations de nos pays. Lorsque l'on laisse entièrement au marché et au secteur privé la tâche d'orienter et de guider les sociétés européennes vers la société mondiale de l'information - comme l'ont fait les dirigeants de l'Union européenne ces cinq dernières années -, c'est non seulement l'État que l'on jette aux orties mais, avec lui, le citoyen lui-même que l'on relègue à un rôle secondaire, subordonné, par rapport au marché, aux entreprises privées et à sa qualité de "consommateur".

L'aspect le plus grave et dangereux dans cette évolution est qu'en devenant les paladins de l'inévitabilité de l'intégration/adaptation compétitive de l'économie nationale dans l'économie mondiale - même si "à visage humain - les pouvoirs publics ne contribuent pas à faire grandir les conditions pour le développement d'un pouvoir politique mondial mais, bien au contraire, contribuent à renforcer le pouvoir privé mondial. II en va de même au plan de la construction européenne. Plus les États membres pratiquent les principes de la libéralisation, de la dérégulation et de la privatisation, plus ils s'éloignent de la construction d'un pouvoir politique fédéral européen. Ils contribuent, en revanche, à l'ultérieure fragmentation politique de l'Europe qui elle, se réduit de plus en plus aux dynamiques du marché (Marché Intégré Unique...). Plus les Européens ont subordonné la politique technologique de l'Europe au soutien des besoins de l'industrie, plus ils ont contribué à diviser les Européens entre eux et à donner à l'industrie le pouvoir de fixer l'ordre du jour du développement technologique, économique et social de nos pays. 
Abandon du système du Welfare. On a démantelé la richesse commune. On a affaiblit les liens sociaux. Le principe de la solidarité en prend un coup sérieux. Où va la citoyenneté sociale?

Le retour massif de la pauvreté dans les pays les plus développés du monde représente non seulement la négation de l'État moderne, mais elle se traduit par le fait que l'économie actuelle se pose en ennemie de la citoyenneté. Plus de 60 millions de pauvres (sur 300 millions) aux ÉtatsUnis, le pays le plus riche et puissant du monde. Plus de 52 millions de pauvres (sur environ 300 millions) dans les pays de l'Union européenne, la plus grande puissance commerciale du monde. Rien qu'au RoyaumeUni, on a enregistré, en 1994, 13,9 millions de pauvres sur environ 60 millions de personnes. Il en va de même de l'exclusion sociale. Le Royaume-Uni est le pays marqué par les plus grandes inégalités de revenu entre individus au monde. Il est suivi par les États-Unis où $1 \%$ de la population possède $39 \%$ de la richesse du pays. Les inégalités dans le revenu disponible ont augmenté de plus de $30 \%$ entre 1981 et 1995 au Royaume-Uni et aux États-Unis.

La technologie est l'un des principaux facteurs des dynamiques d'exclusion sociale dans le contexte de la politique technologique actuelle: une fois qu'un cadre, un ingénieur, un ouvrier, un employé est éjecté du marché du travail, car remplacé par la technologie, il a peu de chances d'y revenir, sauf s'il acquiert de nouvelles compétences supérieures qui lui garantiront, peut-être, un délai de survie en attendant d'être mis en danger par de nouvelles technologies encore plus performantes.

Dans nos sociétés dites "développées", nous n'avons plus grand chose "en commun", mis à part ce qui reste du Welfare State et que l'on cherche précisément à effacer encore davantage. L'économie actuelle est en train de nous faire perdre le sens de "être, faire et vivre ensemble", le sens du «bien commun». La priorité est donnée aux itinéraires individuels (ma formation), aux stratégies de survie individuelle (mon emploi, mon revenu), aux biens individuels (ma voiture, mon personal computen), considérés comme l'expression fondamentale et irremplaçable de la liberté aux régimes privatisés d'assurance-maladie et de pension par capitalisation, au "workfare». On appelle cela, dans certains pays, la politique sociale active.

La logique de la compétitivité a été élevée au rang d'impératif "naturel» de la société et de l'économie où l'aboutissement «inévitable», c'est la victoire de l'un sur l'autre, l'élimination de l'autre. La solidarité est désormais considérée comme un coût insupportable qui pèse lourd, comme un boulet aux pieds, pour les entreprises et leur compétitivité sur 
le marché mondial. Une société qui pense qu'elle n'a pas d'autre choix que le choix, soit de maintenir des chômeurs dans la dépendance d'assistés, soit de mal rémunérer des employés et des ouvriers en appauvrissement croissant, est une société qui a perdu le sens des liens sociaux et du long terme sous l'emprise de la culture du plus fort et de la conquête pour la survie.

En termes économiques, cela signifie qu'il y a eu retournement de position, par rapport aux années ' 50 , '60 et ' 70 , en ce qui concerne les principes fondateurs de la redistribution de la richesse. Désormais, la redistribution des gains de productivité est de nouveau en faveur du capital au détriment du travail. Qu'elles soient néo-libérales ou socialdémocrates, les forces politiques des pays les plus développés de la planète ne se différencient pas beaucoup sur le plan des orientations politiques dans leur support d'une telle redistribution.

Les arguments défendus par les forces qui ont réussi à faire de l'économie du marché capitaliste mondialisée, libéralisée, déréglementée, privatisée, compétitive, l'économie "qui gouverne le monde", sont de nature manifestement idéologique.

II s'agit, d'une part, des thèses affirmant qu'il n'y a pas de droits sociaux acquis, inhérents à la citoyenneté. Pour l'économie de marché, disent-ils, il n'y a pas de citoyenneté sociale reconnue par décret : la citoyenneté s'acquiert et elle peut être enlevée, amputée, suspendue. Tout dépend du niveau de performance et du degré de rentabilité de la "ressource humaine» ainsi que de la bonne performance de l'économie et des disponibilités financières. Si les ressources financières pour les dépenses publiques font défaut, pas de sécurité sociale, pas de citoyenneté sociale, même si les marchés financiers affichent une santé éclatante. En effet, dans l'économie de marché actuelle, c'est précisément le cas : lorsque les marchés financiers se portent bien, c'est logique que la citoyenneté sociale se porte mal.

II s'agit, ensuite, des thèses sur le prétendu échec de l'État du Welfare dans sa lutte pour le plein emploi et contre la pauvreté. C'est parce que l'État du Welfare est fondé sur un vice économique de base "génétique" - disent les arguments - qu'il a échoué dans la réalisation de ses propres finalités constitutionnelles. Ce vice consisterait à ne pas avoir compris que dans toute économie capitaliste - comme est restée l'économie des pays où on a développé le Welfare State - il y a des limites structurelles au niveau possible de taxation. Cet argument me paraît valable à condition que l'on précise que la limite structurelle se situe par rapport au niveau maximum de taxation acceptée et acceptable par le 
capital et non pas par rapport au niveau éventuellement accepté par la société6.

Le largage des villes, des régions, des pays, par cause de guerres économiques mondiales.

On invoque très souvent les progrès technologiques pour expliquer et «justifier» les destructions des tissus économiques et sociaux de régions entières, l'abandon des villes, régions et pays qui ne parviennent pas à réaliser à temps et avec force compétitive la reconversion de leur économie en adaptation aux changements sur l'échiquier économique mondial.

On invoque, également, encore plus souvent, les rigidités dites "sociales" (à savoir, formes avancées de sécurité sociale, régulation du marché du travail, forte présence de syndicats actifs dans le cadre d'une concertation sociale intense...) comme cause principale des délocalisations économiques et du manque d'intérêt des investisseurs "et étrangers" pour des régions et des pays qui tentent de sortir de leur situation et qui, faute d'un tel intérêt, risquent davantage de rester dans un état de "largage» économique.

On fait moins souvent, en revanche, mention des logiques excluantes et ségrégationnistes propres à l'économie actuelle "qui gouverne le marché", pour expliquer pourquoi certaines régions et certains pays entiers tombent progressivement et, parfois, rapidement dans la zone des espaces et des populations qui n'intéressent plus les gestionnaires/organisateurs privés et publics de l'économie mondiale d'aujourd'hui.

Or, comme le confirment les dernières données fournies par la CNUCED, $85 \%$ des investissements directs mondiaux à l'étranger sont destinés, encore une fois, comme tout au long des 15 dernières années, aux pays les plus développés du monde. Autrement dit, les riches investissent chez les riches, ce qui est tout à fait logique dans le cadre de l'économie de marché compétitive, car l'objectif principal d'un investissement financier est d'augmenter les avoirs financiers des possesseurs des capitaux. Les investissements vont là où le taux de profit est le plus élevé et le plus rapide possible. Ils vont donc vers les marchés les plus solvables.

Il est également évident - malgré les dénégations idéologiques des responsables de l'OMC et du FMI - que la libéralisation des marchés et du commerce international à l'enseigne de la compétitivité pour la con-

6. J'ai analysé - sans les partager - les critiques formulées à l'encontre du Welfare State dans R. PETRELLA, Le Bien Commun. Éloge de la solidarité, Bruxelles, Éd. Labor, 1996. 
quête des marchés, obligent les pays les plus pauvres à orienter leur économie vers la production de biens destinés à l'exportation (comme c'est le cas quasi généralisé des politiques de production agricole, alimentaire, textile, énergétique et autres). Cela ne fait qu'augmenter l'appauvrissement de ces pays et amplifier la misère et la faim dans le monde?.

La promotion et la défense du droit de propriété intellectuelle appartient à la même logique: laisser aux forces de marché la possibilité de bréveter à des buts lucratifs des espèces microbiennes, végétales et animales ainsi qu'humaines constitue un autre exemple majeur de la tendance à l'appropriation privée des ressources matérielles et immatérielles de l'humanité.

L'exemple de l'eau est également frappant. Considérée en principe, comme le bien commun patrimonial de l'humanité, l'eau est en train d'être privatisée partout. Le scénario de la "pétrolisation" de l'eau s'affirme de plus en plus rapidement comme un scénario fort probable, si des mesures vigoureuses ne sont prises dans les toutes prochaines années en faveur d'une ré-appropriation de l'eau en tant que res publica mondiale.

II n'y a plus de cadre, d'employé, de travailleur : tous nous sommes devenus des ressources humaines à utilité temporaire et intérimaire.

Le travail a cessé d'être un droit. Il est devenu, ces dernières années, une situation à conquérir. Les responsables de nos économies ne font que répéter que ce n'est plus possible de donner un emploi à tout le monde. Le plein emploi de jadis, affirment-ils, est fini. Et si jamais on devait revenir à des formes de plein emploi, ce serait - dit-on - sur de nouvelles bases et selon des modalités différentes, par exemple par la réduction massive du temps de travail «travailler moins pour travailler tous". Le devoir d'employabilité après la place du droit au travail.

Pourtant, nos sociétés continuent à considérer que pour chacun de nous tout passe par le travail rémunéré, l'accès au revenu, l'intégration sociale, le positionnement dans la société, la satisfaction de se sentir utile et reconnu, l'auto-réalisation.

7. Cf. Le Rapport de la CNUCED de 1999 sur le commerce et les investissements internationaux.

8. Parmi les tenants des thèses en faveur de la marchandisation de l'eau et de la privatisation des services d'eau, figure le Conseil Mondial de l'Eau, une institution créée au milieu des années '90 par la Banque Mondiale. Voir à ce sujet R. PETRELlA, Le Manifeste de l'Eau, Bruxelles, Éd. Labor, 1998, et Comité Promoteur pour le Contrat Mondial de l'Eau (présidé par Mario Soares), Manifeste de l'Eau, Lisbonne, 1997. 
À quoi est dû, dès lors, le décalage entre les valeurs affirmées par la société et le fonctionnement de l'économie qui dit ne pouvoir assurer plus de travail pour tous ${ }^{9}$ ?

Les causes sont nombreuses. Le travail a cessé d'être un droit au fur et à mesure que les principes et les pratiques de l'économie de marché capitaliste libéralisée, déréglementée, privatisée, compétitive ont réussi à s'imposer comme «les Nouvelles Tables de la Loi». C'est dans ce contexte que la personne humaine à été transformée en ressource humaine. Nous ne sommes plus des travailleurs, des maçons, des conducteurs de tram, des paysans, des professeurs d'université, des employés de banque, des assureurs. Nous avons été tous, sans exception, réduits à des ressources humaines appartenant ainsi à l'ensemble des ressources économiques d'un pays et du monde, au même titre que les ressources naturelles, les ressources technologiques, les ressources financières.

En tant que ressource la personne humaine n'a droit à exister que dans la mesure où elle contribue à l'efficacité de la production des biens et des services vendables sur les marchés solvables. Son droit à l'existence cesse de lui être reconnu dès que la ressource humaine n'assure plus l'efficacité dans la mesure souhaitée par l'entreprise, à savoir par ses propriétaires-dirigeants et/ou ses actionnaires: soit parce que son coût est devenu plus élevé que d'autres ressources humaines disponibles ailleurs, soit parce qu'elle est devenue obsolète, soit parce que l'usage d'autres ressources productives assure une plus grande rentabilité. Dans ces conditions, elle est éliminée, jetée à la casse comme toute automobile qui ne fonctionne plus ou tout autre outil qui n'est plus capable d'exercer la fonction pour laquelle il a été conçu et utilisé.

Combien d'ouvriers, de cadres, d'employés, d'ingénieurs n'ont-ils déjà entendu dire qu'ils étaient devenus des ressources humaines dont on ne savait plus quoi faire, et qu'ils n'étaient même plus "recyclables"?

Devenue ressource, la personne humaine n'est plus un sujet social ayant des droits, des envies, des besoins. Elle n'est plus qu'un cout pour l'entreprise à comparer aux autres ressources de l'entreprise selon les critères d'efficacité et de performance (en termes de rentabilité, de profit) fixés et évalués par ceux qui ont le pouvoir de direction et de contrôle de l'activité de production.

9. Encore dix-huit millions de chômeurs en 1998 dans les pays de l'Union Européenne. Plus d'un milliard à l'échelle mondialel Le bas niveau affiché de chómage aux États-Unis cadre une situation de usous"-emploi, d'emploi d'exploitation et d'emploi précaire qui commence à être acceptée et tolérée aussi dans les pays de l'Union européenne. 
Toute autre dimension ou valeur sociale, culturelle, humaine, politique, éthique est subordonnée aux critères d'efficacité et de performance économico-financières. Ces derniers déterminent les cycles de vie des compétences humaines variant désormais entre 3 et 7 ans. Les compétences deviennent vite dépassées, inutiles. Nous sommes dès lors obligés à nous former, à nous recycler sans cesse. Tel est le sens de l'impératif de l'éducation continue pour toute la vie.

Ainsi, dit-on, personne ne peut garantir un emploi dans 5-7 ans car d'ici là tout peut changer et quasi certainement on n'aura plus besoin des savoirs, des métiers et des expériences que nous avons appris et accumulés jusqu'à présent.

La prétention à un travail à durée indéterminée et à plein temps ne serait plus légitime, même dans la fonction publique : en tant que ressource la seule chose à laquelle on peut prétendre c'est un emploi temporaire, de plus en plus à temps partiel, et, bien entendu, dépourvu des garanties sociales qui avaient été associées au droit au travail jusqu'à il y a une dizaine d'années.

En tant que ressource humaine nous devons être très flexibles, malléables, adaptables, transférables, prêts au nomadisme des lieux et des temps, voire même à disparaître (provisoirement?) dans le seul but d'assurer la plus grande compétitivité possible de l'entreprise sur les marchés mondiaux.

\section{VERS UNE NOUVELLE POLITIQUE SOCIALE EUROPÉENNE DANS LE CADRE D'UN WELFARE MONDIAL. DES «RÈGLES DE LA MAISON» ALTERNATIVES SONT POSSIBLES.}

La tendance du pouvoir dominant - et cela même après le rejet en 1998 de l'Accord Multilatéral sur les Investissements (I'AMI) - est de continuer à nous faire croire que la seule politique réaliste possible, face à l'économie actuelle, est de "s'adapter» en transformant en opportunités les "contraintes exogènes" qui seraient représentées par la mondialisation libéralisée, déréglementée, privatisée, compétitive, par les marchés financiers, et par les nouvelles technologies. Drôle de liberté que celle octroyée par le marché mondial !

Délégitimer les principes fondateurs et les priorités de l'économie capitaliste de marché mondiale reste le premier pas à faire, ce que des milliers de mouvements et d'associations à travers le monde ont précisément fait ces dernières années, et qui ont manifesté ouvertement, avec succès, à Seattle. 
L'enjeu de la délégitimation : commencer par d'autres priorités -L'exemple de l'eau

Vers 2020-2025, la population mondiale sera composée de 8 milliards de personnes (6 milliards en 1999), si entre-temps des épidémies, des famines, des guerres n'en auront pas disposé autrement. La véritable question pour l'économie mondiale n'est pas l'intégration/adaptation des économies locales dans l'économie mondiale mais de savoir quels principes, quelles règles et quelles institutions, doivent être définis et mis en œuvre au cours des 25 prochaines années en place et lieu des principes propres à l'économie de marché capitaliste, afin que les 8 milliards de personnes puissent être des sujets citoyens, capables de satisfaire leurs besoins de base en eau potable, logement, alimentation, énergie, santé, éducation, information, transport, communication, expression artistique, participation à la gestion de la communauté. Autrement dit, sur quelles bases et par quels moyens doit-on et peut-on construire la richesse commune mondiale?

Pour cela, il faut avant tout refuser la rhétorique dominante, ses mots-clés, ses symboles. Puisque l'économie de marché mondiale est incapable de créer la richesse commune mondiale, car ce qu'elle désire c'est de promouvoir l'expression continue de la richesse privée, il est légitime et correct de la part du citoyen de lui refuser le pouvoir de gouvernance de l'économie mondiale. II est faux de dire que la compétitivité pour la survie est un atout pour faire grandir la solidarité et la cohésion sociale entre les membres d'un pays et entre les populations et les régions du monde.

Le développement de la richesse commune mondiale passe par la (ré)invention de nouvelles formes $d$ 'économie mutualiste, coopérative, solidaire. Au lieu de libéraliser le "marché» de l'eau et de le soumettre à la dérégulation et à la privatisation, l'eau, ressource considérée depuis toujours, dans toutes les civilisations, comme le bien commun patrimonial de l'humanité, peut et doit devenir l'exemple du premier bien de la richesse commune mondiale géré par des principes coopératifs, mutualistes et solidaires.

Jadis, les "chartes des villes", la "charte des mers", "les chartes des champs", avaient contribué à jeter les bases d'une économie urbaine, marine et rurale plus ou moins coopérative. Aujourd'hui, "sles chartes des rivières", qui engagent toutes les parties concernées vivant autour et des biens et services offerts par une rivière (habitants, exploitants agricoles, entreprises industrielles, associations de diverse nature, autorités publiques aux divers niveaux, exploitants touristiques...) 
à gérer la rivière dans l'intérêt commun, montrant qu'une gouvernance de type coopératif est solidaire d'un bien patrimonial comme l'eau, ces chartes sont, non seulement possibles, mais constituent la base indispensable pour en assurer la gestion efficace et durable.

\section{L'enjeu du désarmement financier.}

Ce qui précède a peu de chance de réussir si, en même temps, on ne procède pas au désarmement de la puissance financière, avec l'aide, en particulier, d'une action coordonnée, à l'échelle mondiale, entre les économistes progressistes des pays du monde développé. À cette fin, les mesures suivantes sont à prendre en priorité :

- prélèvement d'une taxe de $0,5 \%$ sur les transactions financières. Cette mesure a été proposée déjà en 1983 par le Prix Nobel de l'économie, Mr. Tobin. Une telle taxe permettrait de constituer un Fond Mondial de la Citoyenneté doté de plusieurs dizaines de milliards de dollars par an, destinés à financer justement le Contrat Social Mondial, en communiquant par le Contrat de l'Eau et d'autres interventions dans les autres domaines constitutifs de la richesse commune mondiale. Le prélèvement de cette taxe est techniquement faisable. II doit être décidé au niveau du G7 ou du G20 pour surmonter l'argument - qui n'est qu'un alibi - utilisé par chaque pays séparément, consistant à dire qu'il ne peut pas prendre l'initiative d'une telle taxe sans risque de voir tous les capitaux du pays fuir ailleurs;

- élimination des paradis fiscaux. II y a 37 paradis fiscaux de par le monde. Leur existence constitue une forme légalisée de la criminalisation croissante de l'économie (évasion fiscale, spéculation, commerce de la drogue, commerce illicite des armes). Grâce aux paradis fiscaux le monde financier est de plus en plus habité par des prédateurs, dont les entreprises industrielles qui créent de la richesse réelle sont elles-mêmes les victimes. Les vrais industriels n'ont aucun intérêt à ce que le système financier actuel se maintienne et se développe.

Or, que font les gouvernements des pays les plus développés? Au lieu d'éliminer les paradis fiscaux, ils contribuent à leur multiplication, sous forme de création de centres de coordination financière internationale où les holdings financiers multinationaux peuvent se domicilier sans être taxés sur les profits. On assiste à une féroce concurrence entre les pays notamment européens - en matière de facilitations et de réductions fiscales.

- mettre fin au secret bancaire. Le respect du principe de la liberté de propriété et du droit à la confidentialité peut être assuré, sans pour 
autant maintenir le secret bancaire. En outre, une véritable politique fiscale progressiste, fondée sur la justice sociale et la solidarité entre les individus, les générations et les populations des pays de plus en plus interdépendants et intégrés sur le plan économique, passe par l'abolition du secret bancaire

- rendre public et transparent l'évaluation des marchés financiers. Aujourd'hui, il y a trois grandes sociétés privées d'experts financiers qui établissent le classement (le rating) des divers pays du monde, en fonction de ce que ces experts considèrent être la "santé financière" du pays. Ils le font dans le secret de leurs bureaux, sans devoir rendre compte à aucune autorité politique et monétaire. En juillet 1996, suite aux coupes importantes prévues dans les dépenses de l'État, l'Italie s'est vu gratifiée par l'une des trois sociétés financières, l'Américaine Moody, d'une meilleure cotation, passant du niveau A3 à AA1. En Italie, l'avenir de l'un des 7 pays les plus industrialisés du monde dépend du rating qui lui est attribué par une société privée d'experts financiers!

L'ensemble de ces mesures devrait être encadré par la création, à l'échelle mondiale, d'un Conseil Mondial pour la Sécurité Économique et Financière, dont la tâche principale serait de redéfinir les règles d'un nouveau système financier mondial (le "Bretton Woods" pour le XX|e siècle) et de veiller au bon usage de la finance au service du développement et du bien-être de base de l'ensemble de la population mondiale.

\section{L'enjeu de l'innovation technologique et du plein emploi.}

L'usage actuel de la technologie (stratégie de l'offre pour la réduction des coûts de production, l'amélioration de la qualité, l'augmentation de la variété et la croissance de la flexibilité) soulève un problème structurel majeur : c'est elle, en une très large mesure, qui détermine le volume de la demande de travail humain nécessaire pour produire les biens et services dont nous nous entourons. Le volume total du temps de travail humain est devenu le résultat urésiduel» de la technologie. En 1971, il fallait plus de 110 heures de travail humain pour produire une voiture. Aujourd'hui, il n'en faut plus que 14. Dans 10 ans, peut-être 8-9 heures. Jusqu'où nos économies, nos sociétés, laisseront le volume total de temps de travail humain être une variable dépendante de la technologie, tout en considérant que le travail rémunéré reste et restera, pour tout individu, le ticket d'entrée principal dans la société (accès au revenu, positionnement social, utilité sociale, auto-réalisation, biens sociaux)?

Peut-on considérer, dans ces conditions, qu'il soit justifié, correct et possible, d'assurer le plein emploi grâce à une réduction continue de la durée du travail (travailler moins pour travailler plus)? Au 
rythme actuel d'accélération de l'innovation technologique, dans combien d'années il faudra alors procéder à la réduction du temps de travail à 24 heures hebdomadaires et puis à 18 heures, à 12 heures...? Ce faisant, il y aura toujours un nombre important, bien que de plus en plus restreint, de personnes pour qui le travail qu'elles exercent sera plus ou moins stable, bien rémunéré et apprécié, duquel dépendra largement la richesse de nos sociétés, face aux activités "flexibles" moins bien rémunérées et appréciées de la majorité restante de la population «active».

Sommes-nous sûrs que celle-ci soit la bonne voie? Ne faudrait-il pas plutôt songer à re-concevoir le rôle et l'usage que nous donnons à la technologie et à l'innovation technologique? Au lieu d'utiliser la technologie pour gagner des quotes-parts de marché dans les marchés solvables saturés des pays développés, il convient de mettre en place une politique de l'innovation technologique visant à produire des technologies (processus de production, biens et services) destinées à satisfaire les besoins non ou mal satisfaits (la société mondiale n'est point saturée à cet égard) et des nouveaux besoins : tant que la société considère et considérera, comme c'est le cas, que le travail rémunéré reste et restera, pour de nombreuses générations encore, le moyen principal d'intégration dans la société, nos économies ont l'obligation d'assurer le plein emploi pour tout le monde, à l'échelle de la planète. Et c'est à cette fin que la technologie devra être utilisée. Tel est le sens profond de l'enjeu de la productivité pour aujourd'hui et les 20 prochaines années (au moins).

Ceci nous amène au quatrième et dernier enjeu pour l'action.

\section{L'enjeu de la redistribution de la richesse}

Le capital se porte bien. On réduit considérablement la pression fiscale. II peut se balader sans véritables limites et contraintes à travers le monde. Les États lui font des cadeaux pour l'attirer dans leurs pays. On lui confie la tâche de fixer les priorités dans l'allocation des ressources disponibles. On lui offre des ressources humaines de moins en moins chères, flexibles, recyclables.

Le travail, de son côté, se porte mal. La pression fiscale augmente. On lui demande d'être de plus en plus mobile, sans garantie à long terme. On lui demande d'accepter tous les statuts possibles et imaginables pour augmenter sa flexibilité. On lui dit que la technologie le remplacera de plus en plus, inexorablement et qu'il doit se battre, contre les autres, pour garder son emploi à court terme. Le travail s'appauvrit alors que le capital s'enrichit. Le travail perd sa force de négociation. II ne participe plus à la fixation de l'ordre du jour : il est acculé à ré-agir constamment aux décisions prises «ailleurs». 
Cela ne pourra pas continuer toujours. Sans attendre que l'économie de marché n'implose, il est urgent de procéder à une nouvelle redistribution des gains de productivité entre capital et travail en faveur des personnes (les citoyens), et cela à l'échelle mondiale où l'économie actuelle est désormais structurée et opère l'économie.

Cette redistribution passe par une politique du plein emploi axée sur la satisfaction des besoins de base de la population mondiale (encadrant la politique de réduction de temps de travail dans les pays développés saturés) : par la taxe mondiale sur les transactions financières internationales déjà mentionnée; par une politique rigoureuse de gestion de la technologie (moyennant aussi des stimulants fiscaux pour une technologie finalisée à la valorisation des compétences humaines) et des ressources structurelles (éco-taxes à l'échelle mondiale); par une fiscalité progressive efficace (redéfinition des impôts sur la fortune); par la lutte contre l'évasion fiscale et la criminalisation de l'économie; par le renouveau des organisations coopératives et mutualistes.

Bref, les possibilités d'innovations politico-économiques dans ce domaine sont considérables. Ce qui, aujourd'hui, pose problème, à cause des nouvelles Tables de la Loi qui nous gouvernent, c'est l'absence d'un choix "politique" en faveur de la richesse commune et du bien commun. II est temps de définir et mettre en œuvre le Contrat Social Mondial.

\section{Une autre Europe sociale différente de celle prêchée à Lisbonne}

C'est dans le cadre des grandes orientations ci-dessus esquissées qu'une politique sociale européenne peut et doit être considérée et mise en œuvre.

L'Europe sociale redéfinie au récent sommet de Lisbonne présente des bons points, notamment au niveau de la inétorique. À ce niveau, le langage est même captivant : la référence est fréquente aux objectifs de la sécurité pour tous, de l'égalité des chances, du revenu minimum, de la participation des citoyens à la vie sociale, de la lutte contre l'exclusion sociale.

On connaît ces discours depuis notamment le Livre Blanc de la Commission européenne sur "Croissance, Compétitivité, Emploi» (1993) et le Livre Vert de 1994 sur "L'Avenir de la politique sociale européenne».

La pratique des politiques mises en place ces dernières années et les propositions concrètes formulées à Lisbonne constituent une tout autre réalité : au nom d'un réaménagement structurel du Welfare State considéré inévitable - et de la gestion efficace des politiques communes européennes (priorité a été donnée sur toutes les autres politiques à celle 
de l'achèvement du marché intérieur unique et de son fonctionnement), on a procédé à un véritable démantèlement de l'état de la sécurité sociale et du petit peu de politique sociale européenne qui avait été adoptée au bout de très grands efforts.

À Lisbonne, ce sont les principes politico-culturels fondateurs du Welfare State qui ont été enterrés sous l'étiquette de la "politique sociale active".

La narration sous-jacente à la "nouvelle» Europe sociale prêchée à Lisbonne est très vieille en âge. Elle reprend, en fait, les thèses des philosophes-moralistes de la fin du XVIIIe siècle fondateurs de l'économie capitaliste de marché. Elle redonne la priorité à la maximisation de l'utilité individuelle que seul le marché libéralisé et déréglementé (auto-réglé) peut promouvoir et assurer. D'où la ré-affirmation solennelle à Lisbonne, au son des plus fortes trompettes, de l'inévitabilité de l'impératif de la compétitivité tous azimuts et, en particulier, dans les secteurs portés par les nouvelles technologies d'information et de communication. D'où également l'accent mis sur la promotion de la "nouvelle e-économie» supposée permettre un nouveau cycle d'accumulation de capital et le retour de nos économies à des taux élevés de profit. D'où la récupération de la vieille thèse libérale que le marché et la concurrence sont compatibles avec la solidarité et que la liberté du capital est promotrice de solidarité sociale. Dès lors, la "politique sociale active" ne saurait être une politique contre le marché mais une politique pour et au nom du marché.

La force de cette narration réside dans le fait que la plupart des forces progressistes a fait siens les éléments essentiels de cette narration, pour des multiples raisons de nature sociologique, politicoinstitutionnelle et culturelle.

II y a cependant des minorités de personnes et un certain nombre de groupes organisés qui maintiennent vivants le débat et l'élaboration de solutions alternatives. Une "autre narration" de la société, du vivre ensemble et du monde centré sur le droit à la vie pour tous, non seulement au plan européen mais également pour chacun des 8 milliards de personnes qui habiteront la planète vers 2020-2025. À cette fin, l'Europe se doit de définir et mettre en oeuvre en Europe et - par des alliances intercontinentales réellement coopératives - à l'échelle mondiale une nouvelle «ingénierie» politique et socio-économique destinée à promouvoir une nouvelle architecture du système monétaire et financier mondial, le développement de biens communs mondiaux (sorte de "welfare social mondial»), la mise en place d'un service public européen 
de base. Au lieu de donner la priorité à l'objectif de devenir les plus compétitifs au monde en essayant d'imiter et de rattraper le modèle américain - ce qui constitue un choix préjudiciable pour notre devenir -, les Européens devraient s'atteler à re-définir un "futur pour tous". En ce sens, la nouvelle «ingénierie» correspondante est indispensable pour donner la priorité à la promotion de la richesse commune (l'eau pour tous, l'éducation pour tous, la santé pour tous...). En Europe, elle signifie concrètement la lutte contre les privatisations - déjà réalisées - des télécommunications, des assurances, des caisses d'épargne.... et contre la privatisation montante des transports urbains, des chemins de fer, du gaz, de l'électricité, des hôpitaux, de l'éducation, de l'eau... Cela signifie une politique sociale enracinée dans des entreprises de taille petite et moyenne de type coopératif, mutualiste et à finalité sociale. De même il appartient à des entreprises coopératives publiques nationales et européennes (fortement décentralisées) d'assurer la gestion efficace des services et des biens d'intérêts collectifs, grâce notamment à une politique fiscale redistributive capable de réduire la croissance des inégalités sociales entre les ménages au sein des pays de l'Union. Cela signifie également une politique de l'éducation différente de celle appliquée depuis quelques années de plus en plus instrumentalisée et asservie comme on l'a vu plus haut - aux besoins des entreprises désireuses de bénéficier de "ressources humaines" les plus qualifiées, à bon marché, pour "survivre» dans la compétitivité mondiale.

Irréalisme politique? Rêveries d'un chercheur engagé qui n'aurait pas compris que le monde qui compte, qui a le pouvoir, va dans une autre direction? Je n'en suis pas sûr. Ce dont, cependant, je suis sûr, c'est que l'Europe sociale ne pourra s'appeler telle que dans la mesure où nos dirigeants auront compris que gouverner dans et pour la solidarité et l'efficacité sociales ne signifie pas donner au marché la maîtrise de notre devenir.

Riccardo Petrella

Professeur à l'Université Catholique de Louvain

(Belgique)

Président du Groupe de Lisbonne 